\title{
Clinical and radiological evaluation of epilepsy after ischemic cerebrovascular disease
}

\author{
๑ Akçay Övünç Özön', ๑ Ferhat Cüce ${ }^{2}$ \\ 1/stinye University, Liv Hospital, Clinic of Neurology, Ankara, Turkey \\ 2University of Health Sciences Turkey, Gülhane Training and Research Hospital, Clinic of Radiology, Ankara, Turkey
}

\section{Date submitted:}

11.02.2020

Date accepted:

03.03.2020

Online publication date:

15.06.2020

\section{Corresponding Author:}

Ferhat Cüce MD, Istinye University, Liv Hospital, Clinic of Neurology,

Ankara, Turkey

ferhatcuce@hotmail.com

ORCID:

orcid.org/0000-0003-1831-3868

Keywords: Cerebrovascular disease, epilepsy, seizures

\begin{abstract}
Aims: We aimed to evaluate the onset time, seizure type, response to treatment, etiological causes, electroencephalographic, and radiological features of epileptic seizures that occur after ischemic stroke.

Methods: A retrospective evaluation was performed using the data of 2900 patients admitted to our clinic between October 2016 and June 2019 and diagnosed with ischemic stroke. Those who had epileptic seizures within the first 15 days after ischemic cerebrovascular disease were considered as early-onset seizures (EOS), and those who started on or after day 16 were considered as late-onset seizures (LOS).
\end{abstract}

Results: The study was conducted on 46 patients who had epileptic seizures after ischemic stroke. EOS were detected in 28 of the patients (60.9\%) and LOS in 18 (39.1\%). When ischemia etiologies were examined, cardioembolism was found in approximately half of the cases (47.8\%). Secondary generalized tonic-clonic seizure (SGTCS) (52.2\%) and complex partial seizure $(26.1 \%)$ were the most common ones in patients. Monotherapy was performed in 39 patients $(84.8 \%)$ and polytherapy in 7 patients (15.2\%). A focal epileptiform anomaly was the most frequent abnormality in electroencephalography. The most common involvement in both EOS and LOS was observed in the cortical and subcortical areas.

Conclusions: EOS were more frequent after ischemic stroke. Cortical and subcortical involvement was the most common in terms of seizures in radiological examination. The most common seizure type was SGTCS. Monotherapy was the most frequent application in the treatment. The most commonly preferred antiepileptic drug was levetiracetam. Antiepileptic drug therapy was found to be effective.

\section{Introduction}

The most common disabling disease is stroke and it is the third most common cause of death after coronary heart disease and cancer $(1,2)$. Cerebrovascular diseases (CD) take place in the pathogenesis of epilepsy, especially in older ages, and $45 \%$ of epileptic seizures over 60 years of age are a CD (3). Epileptic seizure after hemorrhagic stroke is more common than ischemic stroke (4).

Two or more non-provoked seizures that occurred during the first week after CD were defined as post-stroke epilepsy (PSE) according to the International League Against Epilepsy (ILAE) criteria (5). Seizures that occur in the first 15 days after stroke are classified as early seizures, and those after 16 days are classified as late seizures (5).

Risk factors for developing seizures after CD include hemorrhagic stroke, cardioembolic stroke, cortical location, and multiple cortical involvements (6). In parallel with the increasing elderly population ratio, the increase in ICD patients makes the determination of possible risk factors for the prevention of PSE increasingly important (7).

In our study, we aimed to determine the clinical and radiological features and risk factors of seizures and to evaluate the relationship with seizure prognosis in early and late epilepsy patients after ischemic cerebrovascular disease (ICD). 


\section{Methods}

This retrospective study was approved by the NonInterventional Ethical Committee of Ankara Liv Hospital (no: 001-2019/003). Between October 2016 and June 2019, 2900, in patients who were admitted to the emergency department at the first 24 hours of neurological symptoms, the ischemia was diagnosed retrospectively. The data of the patients were taken from the hospital follow-up files. Patients who were diagnosed with acute ischemic stroke and who had epilepsy for the first time during the first year of follow-up were included in this study. The exclusion criteria included having pre-stroke seizure, hemorrhagic stroke, cerebral venous thrombosis, brain tumor and metastasis, cranial operation, neurological (such as multiple sclerosis) and metabolic abnormalities (hypoglycemia, ketotic or nonketotic hyperglycemic coma, hyponatremia, alcohol intoxication, hypocalcemia) and chronic systemic diseases (liver and kidney failure, cancer). Two or more non-provoked seizures that occurred one week after ICD were defined as PSE according to the ILAE criteria (5). Seizures that occur in the first 15 days after ICD are classified as early seizures, and those that occur after the 16th day are classified as late seizures (5).

Non-contrasted brain computed tomography and magnetic resonance imaging records of the patients, which were obtained within the first 24 hours after admission to the emergency room, were retrospectively evaluated by a neuroradiologist with more than five years of clinical experience. Radiological diagnosis of acute infarction would be made when the lesion was hyperintense on diffusion weighted imaging and was iso or minimal hyperintense on the T2 sequence.

Localization of lesions was recorded as; both cerebral, cerebellar hemispheres and brainstem, cortical-subcortical, basal ganglia (deep gray matter, internal capsule, periventricular white matter), cerebellum and brainstem. Lesion numbers were determined as single or multiple.

The antiepileptic drugs used by the patients were divided into two groups as monotherapy and polytherapy according to the treatment.

The prognosis was evaluated in three groups according to the frequency of seizures after the treatment. These three groups included those without seizures, those with more than $50 \%$ reduction in the frequency of seizures, and those with less than $50 \%$ reduction in the frequency of seizures after the treatment.

\section{Statistical Analysis}

SPPS-16 package program was used for Statistical Analysis. Descriptive statistical methods were used in the evaluation of the data, mean \pm standard deviation was used in the analysis of numerical data, and number $(\mathrm{n})$ and \% expressions were used in the analysis of categorical data. While comparing binary groups with numerical parameters, an independent t-test was used, and a chi-square test was employed to compare categorical data. $p<0.005$ value was considered as statistically significant (8).

\section{Results}

Eighteen of the patients were female (39.1\%), and 28 were male $(60.9 \%)$. The mean age of the patients was $63.72 \pm 13.50$ years (29-90 years). There was no difference between genders in terms of mean ages (Table 1). Early-onset seizures (EOS) were detected in $28(60.9 \%)$ of 46 patients with ischemic stroke in the study, and 18 late-onset seizures (LOS) were detected $(39.1 \%)$. While the rates of men and women were equal in those with EOS, $77.8 \%(n=14)$ of patients with LOS were men. There was no statistical difference between genders in terms of seizure onset time $(p=0.056)$.

When ischemia etiologies were examined, cardiac embolism was found in approximately half of the cases $(n=22,47.8 \%)$. While large arterial atherothrombosis was present in $32.6 \%$ $(n=15)$ of the cases, $19.6 \%(n=9)$ had small artery occlusion.

When the risk factors of the patients were evaluated, hypertension (HT) was detected in 29 (63.0\%) patients, atrial fibrillation in $22(47.8 \%)$ patients and diabetes mellitus in 20 $(43.5 \%)$ patients. The smoking rate was $30.4 \%(n=14)$ and the rate of hypercholesterolemia was $28.3 \%(n=13)$.

Considering the localization of the lesions, while the most common involvement in both EOS and LOS was in the corticalsubcortical area $(78.6 \%$ and $72.2 \%$, respectively), LOS were found more in those with basal ganglion involvement, and EOS in those with cerebellum involvement. There was no statistical difference between localization and time of onset of seizures.

When the number of ischemic lesions was examined, there was more than one lesion, especially in patients with LOS $(p=11$, $61.1 \%$ ). Ischemic lesions were present in both hemispheres of the brain in $17.4 \%(n=8)$ of the patients. In more than half of the patients with EOS, the lesion was on the unilateral right side $(n=16,57.1 \%)$.

While secondary generalized tonic-clonic seizure (SGTCS) $(52.2 \%)$ and complex partial seizure $(26.1 \%)$ were the most common types in patients, followed by simple partial seizure $(8.7 \%)$ and non-convulsive status epilepticus (SE) $(8.7 \%)$, the most common seizure type in patients with EOS was SGTCS, and it constitutes $60.7 \%$ of seizure types. There was no statistically significant difference between seizure onset time and seizure types $(p=0.479)$.

The most common finding in electroencephalogram (EEG) was focal epileptic abnormality (FEA) (45.7\%). EEG results of 5 patients were evaluated as normal. The most common EEG finding in patients with EOS was FEA (53.6\%), while the most common EEG finding in LOS was focal slow wave. No significant 


\begin{tabular}{|c|c|c|c|c|c|c|c|c|}
\hline \multirow{3}{*}{ Variables $(n=46)$} & & \multicolumn{6}{|c|}{ Seizure time } & \multirow{3}{*}{$\mathbf{p}$} \\
\hline & & \multicolumn{2}{|c|}{ EOS $(n=28)$} & \multicolumn{2}{|c|}{$\operatorname{LOS}(n=18)$} & \multicolumn{2}{|c|}{ Total $(n=46)$} & \\
\hline & & $\mathbf{n}$ & $\%$ & $\mathbf{n}$ & $\%$ & $\mathbf{n}$ & $\%$ & \\
\hline \multirow{2}{*}{ Gender } & Female & 14 & 50.0 & 4 & 22.2 & 18 & 39.1 & \multirow{2}{*}{0.056} \\
\hline & Male & 14 & 50.0 & 14 & 77.8 & 28 & 60.9 & \\
\hline \multirow{3}{*}{ Etiology } & Atherothrombosis & 9 & 32.1 & 6 & 33.3 & 15 & 32.6 & \multirow{3}{*}{0.923} \\
\hline & Small arteries occlusion & 6 & 21.4 & 3 & 16.7 & 9 & 6.19 & \\
\hline & Cardiac embolism & 13 & 46.4 & 9 & 50.0 & 22 & 47.8 & \\
\hline \multirow{4}{*}{ Localization } & Cortical-subcortical & 22 & 78.6 & 13 & 72.2 & 35 & 76.1 & 0.622 \\
\hline & Basal ganglia & 6 & 21.4 & 7 & 38.9 & 13 & 28.3 & 0.199 \\
\hline & Brainstem & - & - & 1 & 5.6 & 1 & 2.2 & 0.207 \\
\hline & Cerebellum & 6 & 21.4 & 1 & 5.6 & 7 & 15.2 & 0.144 \\
\hline \multirow{3}{*}{ Side } & Right & 16 & 57.1 & 8 & 44.4 & 24 & 52.2 & \multirow{3}{*}{0.594} \\
\hline & Left & 7 & 25.0 & 7 & 38.9 & 14 & 30.4 & \\
\hline & Bilateral & 5 & 17.9 & 3 & 16.7 & 8 & 17.4 & \\
\hline \multirow{2}{*}{ Number of lesions } & Single & 14 & 50.0 & 7 & 38.9 & 21 & 45.7 & \multirow{2}{*}{0.551} \\
\hline & More than one & 14 & 50.0 & 11 & 61.1 & 25 & 54.3 & \\
\hline \multirow{4}{*}{ EEG result } & Normal & 3 & 10.7 & 2 & 11.1 & 5 & 10.9 & \multirow{4}{*}{0.659} \\
\hline & FSW & 8 & 28.6 & 8 & 44.4 & 16 & 34.8 & \\
\hline & csW & 10 & 35.7 & 5 & 27.8 & 15 & 32.6 & \\
\hline & FEA & 15 & 53.6 & 6 & 33.3 & 21 & 45.7 & \\
\hline \multirow{5}{*}{ Seizure type } & SGTCS & 17 & 60.7 & 7 & 38.9 & 24 & 52.2 & \multirow{5}{*}{0.479} \\
\hline & CPN & 7 & 25.0 & 5 & 27.8 & 12 & 26.1 & \\
\hline & SPS & 1 & 3.6 & 3 & 16.7 & 4 & 7.8 & \\
\hline & NCSE & 2 & 7.1 & 2 & 11.1 & 4 & 7.8 & \\
\hline & SE & 1 & 3.6 & 1 & 5.6 & 2 & 4.3 & \\
\hline \multirow{2}{*}{ Treatment } & Monotherapy & 25 & 89.3 & 14 & 77.8 & 39 & 84.8 & \multirow{2}{*}{0.407} \\
\hline & Polytherapy & 3 & 10.7 & 4 & 22.2 & 7 & 15.2 & \\
\hline \multirow{4}{*}{ Medication } & LEV & 21 & 75.0 & 8 & 44.4 & 29 & 63.0 & 0.036 \\
\hline & $\mathrm{CBZ}$ & 6 & 21.4 & 6 & 33.3 & 12 & 26.1 & 0.370 \\
\hline & VA & 3 & 10.7 & 2 & 11.1 & 5 & 10.9 & 0.966 \\
\hline & OXC & 1 & 3.6 & 6 & 33.3 & 7 & 15.2 & 0.006 \\
\hline \multirow{5}{*}{ Risk factors } & HT & 20 & 71.4 & 9 & 50.0 & 29 & 63.0 & 0.142 \\
\hline & DM & 11 & 39.3 & 9 & 50.0 & 20 & 43.5 & 0.474 \\
\hline & $\mathrm{HL}$ & 8 & 28.6 & 5 & 27.8 & 13 & 28.3 & 0.953 \\
\hline & SM & 8 & 28.6 & 6 & 33.3 & 14 & 30.4 & 0.732 \\
\hline & AF & 13 & 46.4 & 9 & 50.0 & 22 & 47.8 & 0.813 \\
\hline \multirow{3}{*}{ Prognosis } & Seizure-free & 21 & 75.0 & 10 & 55.6 & 31 & 67.4 & \multirow{3}{*}{0.068} \\
\hline & Seizure decreased by more than $50 \%$ & 3 & 10.7 & 7 & 38.9 & 10 & 21.7 & \\
\hline & Seizure decreased by less than $50 \%$ & 4 & 14.3 & 1 & 5.6 & 5 & 10.9 & \\
\hline
\end{tabular}

EOS: Early-onset seizure, LOS: Late-onset seizure, FSW: Focal slow wave, CSW: Common slow waves, FEA: Focal epileptic abnormality, SGTCS: Secondary generalized tonic-clonic seizure, CPS: Complex partial seizure, SPS: Simple partial seizure, SE: Status epilepticus, NCSE: Non-convulsive SE, LEV: Levetiracetam, CBZ: Carbamazepine, VA: Valproic acid, OXC: Oxcarbazepine, HT: Hypertension, DM: Diabetes mellitus, HL: Hyperlipidemia, SM: Smoking, AF: Atrial fibrillation 


\begin{tabular}{|c|c|c|c|c|c|}
\hline Medicines & The number & ugs & $n$ & $\%$ & $p$ \\
\hline \multirow{4}{*}{ LEV } & \multirow{2}{*}{$\begin{array}{l}\text { Monotherapy } \\
(n=39)\end{array}$} & No & 17 & 43.6 & \multirow{4}{*}{0.028} \\
\hline & & Yes & 22 & 56.4 & \\
\hline & \multirow{2}{*}{$\begin{array}{l}\text { Polytherapy } \\
(n=7)\end{array}$} & No & - & - & \\
\hline & & Yes & 7 & 100.0 & \\
\hline \multirow{4}{*}{ CBZ } & \multirow{2}{*}{ Monotherapy } & No & 30 & 76.9 & \multirow{4}{*}{0.272} \\
\hline & & Yes & 9 & 23.1 & \\
\hline & \multirow{2}{*}{ Polytherapy } & No & 4 & 57.1 & \\
\hline & & Yes & 3 & 42.9 & \\
\hline \multirow{4}{*}{ VA } & \multirow{2}{*}{ Monotherapy } & No & 36 & 92.3 & \multirow{4}{*}{0.102} \\
\hline & & Yes & 3 & 7.7 & \\
\hline & \multirow{2}{*}{ Polytherapy } & No & 5 & 71.4 & \\
\hline & & Yes & 2 & 28.6 & \\
\hline \multirow{4}{*}{ OXC } & \multirow{2}{*}{ Monotherapy } & No & 34 & 87.2 & \multirow{4}{*}{0.285} \\
\hline & & Yes & 5 & 12.8 & \\
\hline & \multirow{2}{*}{ Polytherapy } & No & 5 & 71.4 & \\
\hline & & Yes & 2 & 28.6 & \\
\hline
\end{tabular}

difference was found in seizure onset time among patients with EEG abnormalities ( $p=0.659)$.

Monotherapy was performed in 39 patients $(84.8 \%)$ and polytherapy in 7 patients $(15.2 \%)$. Three $(10.7 \%)$ of patients undergoing polytherapy had EOS, and 4 (22.2\%) had LOS. There was no significant relationship between the number of drugs used and the seizure of EOS or LOS $(p=0.407)$ (Table 2).

When the drugs used by the patients were examined, it was observed that levetiracetam (LEV) $(63.0 \%)$ was used mostly. In patients using a single medication, $56.4 \%(n=22)$ used LEV while all patients receiving polytherapy had LEV usage.

Seizure recurrence was not observed in 31 of the patients $(67.4 \%)$. While more than $50 \%$ reduction in seizures of 10 patients $(21.7 \%)$ were achieved, improvement in seizure recurrence of 5 patients (10.9\%) was less than $50 \%$.

\section{Discussion}

This study showed that different types of epileptic seizures occur as a complication of ICD. Cardiac embolism and atherothrombosis were found to be the most common etiology of ICD, and HT was the most common risk factor. Epileptic seizures were frequently associated with ischemic involvement with cortical-subcortical localization. Focal seizures were the most common after ICD.

In large epidemiological studies from the literature, the most common cause of partial and generalized seizure etiology has been shown as cerebrovascular disease (9). It showed different rates, such as $35 \%, 48 \%$, and $54 \%$ for early seizures after stroke (10-12). In one study, the rate of seizures seen in a year after stroke was $3.4 \%$, while $56 \%$ of patients had an early onset partial character and $72 \%$ of late seizures were observed as generalized tonic-clonic features (13). In our study, EOS were detected in $28(60.9 \%)$ of 46 patients with ischemic stroke, and late-onset were detected in 18 (39.1\%).

In a study by Barańska-Gieruszczak et al. (14), 483 cases who had epileptic seizures after stroke were evaluated and it was reported that early seizures were commonly generalized and less frequently simple partial. In our study, the most common seizure type in those with EOS was SGTCS.

In the study of Burneo et al. (15), no statistically significant difference was found between the presence of seizures and age. In our study, there was no statistically significant difference in terms of gender $(p=0.056)$, seizure onset time, and age (0.690).

Although they did not find a significant relationship between cortical involvement and EOS in the Copenhagen stroke study, some authors showed a significant relationship between EOS and cortical involvement (16). In our study, considering the localization of the lesions, while the most frequent involvement in both EOS and LOS was in the cortical-subcortical area $(78.6 \%$ and $72.2 \%$, respectively), LOS were found more in those with basal ganglion involvement, and EOS were more common in those with cerebellum involvement.

It was reported in the literature that SE after serious stroke was more common, and in the early period (17). In our study, the SE case was seen at both one early and one in the late period.

While the prognosis of epileptic seizures developing after a stroke is well accepted, the response to monotherapy is generally good. In the literature, this rate was reported to be $88 \%$ in one study (18). In our study, 39 (84.8\%) patients were treated with monotherapy, 7 (15.2\%) were treated with polytherapy, and $31(67.4 \%)$ of them had no recurrence of seizures during the follow-up.

LEV, which is one of the new antiepileptics, has been the most preferred antiepileptic in terms of side effects and tolerability considering the patient's average age. In our study, the most commonly used antiepileptic drug was LEV (63.0\%), while carbamazepine and valproate were preferred less.

In our study, the group evaluated was intended to be more homogeneous, and we have evaluated ischemic stroke and seizure association from the cerebrovascular disease group. The limitations of this study were as follows; the study was retrospective, and the number of patients was small.

Seizures may be seen in the early and late periods associated with ICD. Although the seizures observed during the course of ICD are frequently focal seizures, SE (convulsive and nonconvulsive) with high mortality and morbidity can also be seen. Generally, ICD patients are from the elderly population. Early 
diagnosis of epileptic seizures and rapid initiation of appropriate treatment are very important in terms of prognosis.

Nevertheless, there were some limitations to our study. Firstly, this retrospective study may lead to the possibility of selection bias, and the lack of a control group was limiting the strength of the current analysis. Secondly, the study population was small, and a larger sample size could be better to find the prognosis of PSE.

\section{Conclusion}

As a result, in societies where life expectancy is longer and the elderly population is increased, the importance of ICD related seizures has increased due to its effect on mortality and morbidity. Some data obtained from the studies were found different from each other. These differences may be due to the study design, selected patient subgroups, and non-standard parameters examined. Randomized, double-blind, placebocontrolled, long-term, multicenter studies with large patient groups are needed to fully reveal the frequency, types, treatment responses, and prognosis of epileptic seizures after ICD.

\section{Ethics}

Ethics Committee Approval: This retrospective study was approved by the Non-Interventional Ethical Committee of Ankara Liv Hospital (no: 001-2019/003).

Informed Consent: Retrospective study.

Peer-review: Externally and internally peer-reviewed.

\section{Authorship Contributions}

Concept: A.Ö.Ö., Design: A.Ö.Ö., Data Collection or Processing: A.Ö.Ö., Analysis or Interpretation: A.Ö.Ö., F.C., Literature Search: A.Ö.Ö., F.C., Writing: A.Ö.Ö., F.C.

Conflict of Interest: No conflict of interest was declared by the authors.

Financial Disclosure: The authors declared that this study received no financial support.

\section{References}

1. Adams RD, Victor M, Ropper HA. Principles of neurology. 8th ed. New York: McGraw Hill; 2006:660-747.

2. Ralph L. Patogenenesis, classification, and epidemiology of the cerebrovascular disease. 10th ed In: Rowland PL, Merritt'sneurology. 2000:217-274.

3. Forsgren L, Bucht G, Eriksson S, Bergmark L. Incidence and clinical characterization of unprovoked seizures in adults: a prospective population-based study. Epilepsia. 1996;37:224-229.

4. Dakaj N, Shatri N, Isaku E, Zeqiraj K. Symptomatic Epilepsies due to Cerebrovascular Diseases. Mater Sociomed. 2014;26:395-397.

5. Guidelines for epidemiologic studies on epilepsy. Commission on Epidemiology and Prognosis, International League Against Epilepsy. Epilepsia. 1993;34:592-596.

6. Cheung CM, Tsoi TH, Au-Yeung M, Tang AS. Epileptic seizure after stroke in Chinese patients. J Neurol. 2003;250:839-843.

7. Conrad J, Pawlowski M, Dogan M, Kovac S, Ritter MA, Evers S. Seizures after cerebrovascular events: risk factors and clinical features. Seizure. 2013;22:275-282.

8. Sonkaya AR, Bayazit ZZ. Language Aspects of Patients with Multiple Sclerosis. EJMI. 2018;2:133-138.

9. Wang G, Jia H, Chen C, et al. Analysis of risk factors for first seizure after stroke in Chinese patients. Biomed Res Int. 2013;2013:702871.

10. Berges S, Moulin T, Berger E, Tatu L, Sablot D, Challier B, Rumbach L. Seizures and epilepsy following strokes: recurrence factors. Eur Neurol. 2000;43:3-8.

11. Demir T, Aslan K, Balal M, Bozdemir H. Clinical Features of Postroke Epilepsy and Relationship with Prognosis. Epilepsi. 2013;19:121-126.

12. Sıvacı AÖ, Örün $M O$, Demir $A B$, Bora İ. Correlation between Lesion Location and EEG Findings in Post-stroke Epilepsy. Epilepsi. 2015;21:20-24.

13. Cheung CM, Tsoi TH, Au-Yeung M, Tang AS. Epileptic seizure after stroke in Chinese patients. J Neurol. 2003;250:839-843.

14. Barańska-Gieruszczak $M$, Romaniak $A$, Ryglewicz D, Niedzielska K, Członkowska A. [Epileptic seizures in poststroke patients]. Neurol Neurochir Pol. 1999;33:815-823.

15. Burneo JG, Fang J, Saposnik G; Investigators of the Registry of the Canadian Stroke Network. Impact of seizures on morbidity and mortality after stroke: a Canadian multi-centre cohort study. Eur J Neurol 2010;17:52-58.

16. Conrad J, Pawlowski M, Dogan M, Kovac S, Ritter MA, Evers S. Seizures after cerebrovascular events: risk factors and clinical features. Seizure. 2013;22:275-282.

17. Verellen RM, Cavazos JE. Pathophysiological considerations of seizures, epilepsy, and status epilepticus in the elderly. Aging Dis. 2011;2:278-285.

18. Silverman IE, Restrepo L, Mathews GC. Poststroke seizures. Arch Neurol. 2002;59:195-201. 